\section{Novel oral anticoagulants: too good to be true?}

\section{Michael Schachter}

It is fair to say that the pharmacological therapy of cardiovascular disease has not been an area of spectacular growth in recent years. Little has happened in the treatment of hypertension and heart failure. There has been interest in the development of antiplatelet drugs in acute coronary syndromes and percutaneous interventions, but not in long-term cardiovascular prevention to compete with aspirin and clopidogrel. The one major area of intense interest has been the novel oral anticoagulants (NOACs), dabigatran, rivaroxaban and apixaban. The first of these is a direct thrombin inhibitor, while the other two are blockers of activated factor X. Two comprehensive reviews of these drugs are published in this issue of the Postgraduate Medical Journal. $^{1}{ }^{2}$ Despite their relatively high costs these drugs have been adopted widely in developed countries for the treatment of venous thromboembolic disease and especially for preventing stroke in atrial fibrillation. This represents a population of several hundred thousand in the UK alone and has been the greatest single driver for developments of the NOACs. The disadvantages of warfarin are well-known; the need for indefinite monitoring; the large interindividual variations in response, largely determined by genetic variability in metabolism; the delay in onset of action; the presence of many interactions through the cytochrome P450 (CYP450); and some idiosyncratic adverse effects, such as necrosis of the skin.

A research objective has been to find a substitute for warfarin that is safer and as effective. As the two reviews indicate, the consensus has been that the new drugs are as efficacious as warfarin, as safe as warfarin as measured by the risk of major haemorrhages, the interactions are fewer, and importantly, there is little variability in response between patients and, hence, no need for plasma level monitoring. As they act directly on clotting factors their onset of action is rapid. The main perceived drawback, admittedly a major one, is the lack of any specific antidote for any of these drugs, as vitamin $\mathrm{K}$ is for warfarin.

Dabigatran was the first of the new drugs to be approved, and this process was

Correspondence to Dr Michael Schachter, Faculty Education Office, Imperial College, Sir Alexander Fleming Building, London SW7 2AZ, UK; m.schachter@imperial.ac.uk expedited by the US Federal Drug Administration (but not by the European Medicines Agency) before the full analysis of the RE-LY trial, which was the key study for regulators. However, recent investigations published in the BMJ (after the present reviews were written and accepted) have cast serious doubt on the claims that the drug shows little interindividual variation in plasma levels, and that monitoring of plasma levels is not relevant for minimising the risk of bleeding. ${ }^{3-5}$ It is particularly worrying that the manufacturer of dabigatran, Boehringer Ingelheim, did not share certain analyses with the regulators suggesting that adjustment of dosage based on plasma levels could reduce the risk of major haemorrhage by up to $40 \%$ as compared with wellcontrolled warfarin without any impact on the risk of ischaemic stroke. The incomplete nature of the evidence on which guidelines for this drug have been based was only disclosed in the course of litigation in the USA and by an investigation by the BMJ. Boehringer Ingelheim explained that such analyses were not shared with the regulators because the analyses did not provide a reliable prediction of patient outcomes, and the research did not support making dosage decisions based on plasma outcomes. Although regular monitoring of this drug would provide clear benefits to patients, it would also reduce any apparent advantage of the drug over warfarin, thus reducing this particular drug's 'selling point'. At the time of writing it is not clear what the regulatory consequence of these revelations might be. Are we likely to discover similar problems with rivaroxaban and apixaban? At the moment we do not know. But in view of their pharmacokinetic properties there may be grounds for cautious optimism. Dabigatran has multiple basic disadvantages: it has very poor bioavailability, 3-7\%, even worse than the bisphosphonates; it is a prodrug which needs two steps to convert it into the active compound; and it is eliminated entirely through the kidneys. All these factors are almost guaranteed to maximise variability in individual pharmacokinetics, and they do not apply to the other drugs. They have bioavailabilities of $50-80 \%$ and are cleared by both liver and kidneys. But it would be reassuring to have all relevant analyses in the public domain.

There is little doubt that the NOACs will have a significant role in anticoagulation- notably, in stroke prevention in atrial fibrillation. But we are not yet confident that we can use them with maximal efficacy and especially maximal safety. It is not clear why dabigatran licensing was expedited in the USA. Everyone agrees that warfarin is far from ideal but it is effective and supported by decades of experience. This situation is hardly comparable to the treatment of cancers or infections where established treatments are ineffective and new treatments urgently need to be developed and tested. In the present situation, obligatory plasma level monitoring of dabigatran should surely be considered by the regulators and the manufacturer. But most disappointing, despite experiences of late withdrawals from use of drugs such as cerivastatin and rofecoxib, ${ }^{6} 7$ the complete disclosure of all relevant analyses does not always happen. It certainly should.

\section{Competing interests None.}

Provenance and peer review Commissioned; internally peer reviewed.

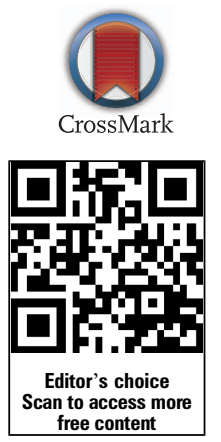

To cite Schachter M. Postgrad Med J 2014;90:485.

Received 4 August 2014

Accepted 7 August 2014

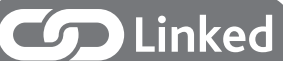

- http://dx.doi.org/10.1136/postgradmedj-2013-132474 http://dx.doi.org/10.1136/postgradmedj-2014-132605

Postgrad Med J 2014;90:485

doi:10.1136/postgradmedj-2014-132950

\section{REFERENCES}

1 Cowell RPW. Direct oral anticoagulants: integration into clinical practice.. Postgrad Med J 2014;90:529-39.

2 Saraf K, Morris P, Garg P, et al. Non-vitamin K antagonist oral anticoagulants (NOACs): clinical evidence and therapeutic considerations. Postgrad Med J 2014;90:520-8.

3 Cohen D. Dabigatran: how the drug company withheld important analyses. BMJ 2014;349:g4670.

4 Moore TJ, Cohen MR, Mattison DR. Dabigatran, bleeding and the regulators. BMJ 2014;349:g4517.

5 Charlton B, Redberg R. The trouble with dabigatran. BMJ 2014;349:g4681.

6 Farmer JA. Learning from the cerivastatin experience. Lancet 2001;358:1383-5.

7 Dieppe PA, Ebrahim S, Martin RM, et al. Lessons from the withdrawal of rofecoxib: observational studies should not be forgotten. BMJ 2004;329:867-8. 